\title{
Psychotherapie im Mausmodell
}

\author{
Was bei EMDR gegen PTBS im Gehirn passiert
}

\author{
Korrespondenzadresse \\ Prof. Dr. Dr. Manfred Spitzer \\ Universität Ulm \\ Abteilung für Psychiatrie \\ Leimgrubenweg 12-14 \\ 87054 Ulm \\ Bibliografie \\ DOI https://doi.org/10.1055/a-0847-8494 \\ Nervenheilkunde 2019; 38: 231-239 \\ (c) Georg Thieme Verlag KG Stuttgart · New York \\ ISSN 0722-1541
}

Zusammenfassung

In einem Mausmodell der Angstkonditionierung und des Verlernens der Angst durch alternierende bilaterale Stimulation (ABS) wurde dem Wirkungsmechanismus der mittlerweile gut etablierten Therapieform EMDR (Eye Movement Desensitization and Reprocessing) zur Behandlung der posttraumatischen Belastungsreaktion (PTBS) nachgegangen. Hierdurch wurde nicht nur gezeigt, dass alternierende bilaterale Stimulation eine notwendige Bedingung für eine schnellere und länger anhaltende Wirksamkeit des Verlernens von Angst im Vergleich zum reinen Extinktionslernen (ohne ABS) darstellt, sondern auch, dass es sich um eine hinreichende Bedingung handelt (u. a. durch Simulation der ABS mittels optogenetischer Stimulation des Colliculus superior). Das damit vorliegende kausale Modell erscheint vor allem für die weitere Erforschung der Mechanismen von Psychotherapie im Tiermodell und für die Hypothesengenerierung bei Forschung am Menschen bedeutsam.
Man mag es zunächst einfach nicht glauben: In der Ausgabe des Fachblatts Nature vom 24. Februar 2019 wurde ein Mausmodell für eine vor allem bei posttraumatischer Belastungsstörung (PTBS) durchgeführten Psychotherapie berichtet, die von Skeptikern bis heute eher belächelt wird. Sie hat den etwas längeren Namen Eye Movement Desensitization and Reprocessing, weswegen man sie nahezu immer mit der Abkürzung EMDR benennt. Man weiß nicht, was man an der Studie mehr bewundern soll: Das kreative Tiermodell des Psychotherapieverfahrens ( $\bullet$ Abb. 1), das ganze Arsenal neurobiologischer Techniken, das zur Anwendung kam oder den Mut, sich die ganze Arbeit überhaupt zu machen. Auf jeden Fall ist die Untersuchung sowohl psychiatrisch als auch neurobiologisch sehr interessant, könnte zukunftsweisend sein und liegt thematisch mitten in der Nervenheilkunde - dem Fach und dem Fachblatt! Zudem lassen die neuen Erkenntnisse die Ergebnisse einer unserer Ulmer Arbeitsgruppen zur funktionellen Bildgebung bei gesunden Versuchspersonen zur Extinktion mittels EMDR in einem neuen Licht erscheinen.

Das Maus-Modell des Extinktionslernens mit und ohne EMDR bestand darin, dass die Tiere nach der Konditionierung nur den Ton ohne elektrischen schmerzhaften Reiz hörten. In einer zweiten Bedingung hörten sie den Ton zusammen mit gleichzeitiger (durch die LED-Lampen bewirkten) Bewegung der Augen. Hierzu wurden die in Blickrichtung des Tiers liegenden 7 LEDs nacheinander von links nach rechts und dann zurück (etwa mit einer Frequenz von $1 \mathrm{~Hz}$ ) eingeschaltet (stark modifiziert nach [5], S. 340, > Fig. 1a sowie nach [17], > Fig. 1).

\section{Zur Geschichte der EMDR}

Die Kalifornierin Francine Shapiro ging an irgendeinem Tag des Jahres 1987 problembeladen im Wald spazieren, achtete dabei mit bewegtem Blick auf ihre Umgebung - und fühlte sich hinterher besser und weniger ängstlich. Dies war für sie so beeindruckend, dass sie bei therapeutischen Gesprächen mit Klienten diese aufforderte, ihre Augen hin und her zu bewegen - und hatte mit dieser Methode Erfolg. ${ }^{1}$ Mittlerweile werden Geräte zum Erwerb angeboten, die den Zeigefinger des Therapeuten durch ein buntes bewegtes LED-Lichtband ersetzen (Farbe und Geschwindigkeit einstellbar, drahtlos per App oder - old school - mit Drähten verbunden steuerbar; Abb. 2).

Trotz der bei der Geburt psychotherapeutischer Verfahren meist üblichen erheblichen Geburtswehen mit Streit über alles Mögliche, von Urheberschaft über Wirksamkeit bis zum Wirkungsme-

1 Entgegen manchen Darstellungen ist Frau Shapiro weder Literaturwissenschaftlerin noch Psychologin, sondern hat nach einem Bachelor im Fachgebiet Englische Literatur zwar ihr Masterstudium in Psychologie mit einer Dissertation beendet - jedoch an einer nicht mehr existierenden und nie akkreditierten Einrichtung („Professional School of Psychological Studies“). Auch der von ihr berichtete Kontext der Entdeckung (Waldspaziergang) wurde von einem der Begründer des neurolinguistischen Programmierens (NLP), John Grinder, verneint. Er behauptet, Frau Shapiro die Sache mit den Augenbewegungen in den 1980er-Jahren beigebracht zu haben, während sie in seinem Büro als Verwaltungsangestellte arbeitete [9]. Dies mag sein, aber das, was man im Bereich der NLP zu Augenbewegungen behauptet hatte, hielt der empirischen Überprüfung ebenso wenig stand, wie diese insgesamt „ungewöhnliche" Form von Psychotherapie). 


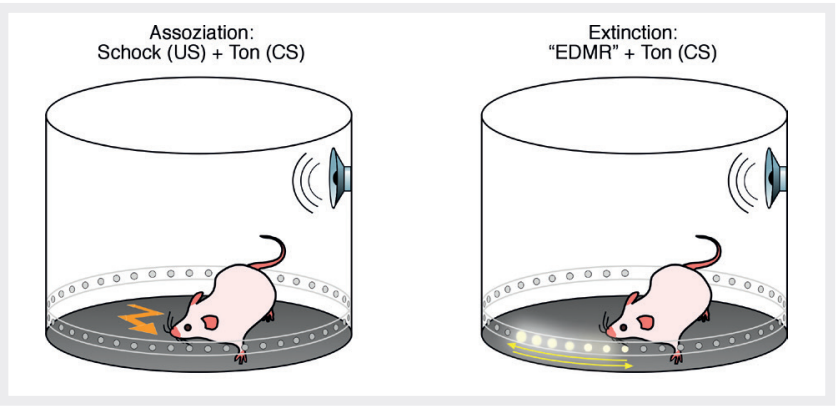

- Abb. 1 Maus-Modell der PTBS (links) und der EMDR (rechts). Der Zylinder bestand aus Acrylglas und hatte einen Durchmesser und eine Höhe von je $20 \mathrm{~cm}$. Der LED-Ring befand sich in $5 \mathrm{~cm}$ Höhe vom Boden entfernt. Wird dem Versuchstier ein schmerzhafter (aber als „milde“ bezeichneter) elektrischer Stromstoß (unkonditionierter Reiz, im Englischen: „unconditioned stimulus“, US) über den elektrisch leitenden Boden des Käfigs („Elektro-Schock“) appliziert, so reagiert es mit einer sichtbaren Schockstarre. Wird der Stromstoß in der letzten Sekunde von einem Ton von $3000 \mathrm{kHz}$ von $90 \mathrm{~dB}$ Lautstärke für 30 s (konditionierter Reiz, „conditioned stimulus“, CS) begleitet, lernt das Tier die Verbindung zwischen dem Ton und dem elektrischen Schock, reagiert also auch nur auf den Ton mit Schockstarre. Dieses Phänomen nennt man seit Pawlow einen bedingen Reflex („conditioned response“, CR). Quelle: Zeichnung nicht genau maßstabsgerecht $\odot$ Autor

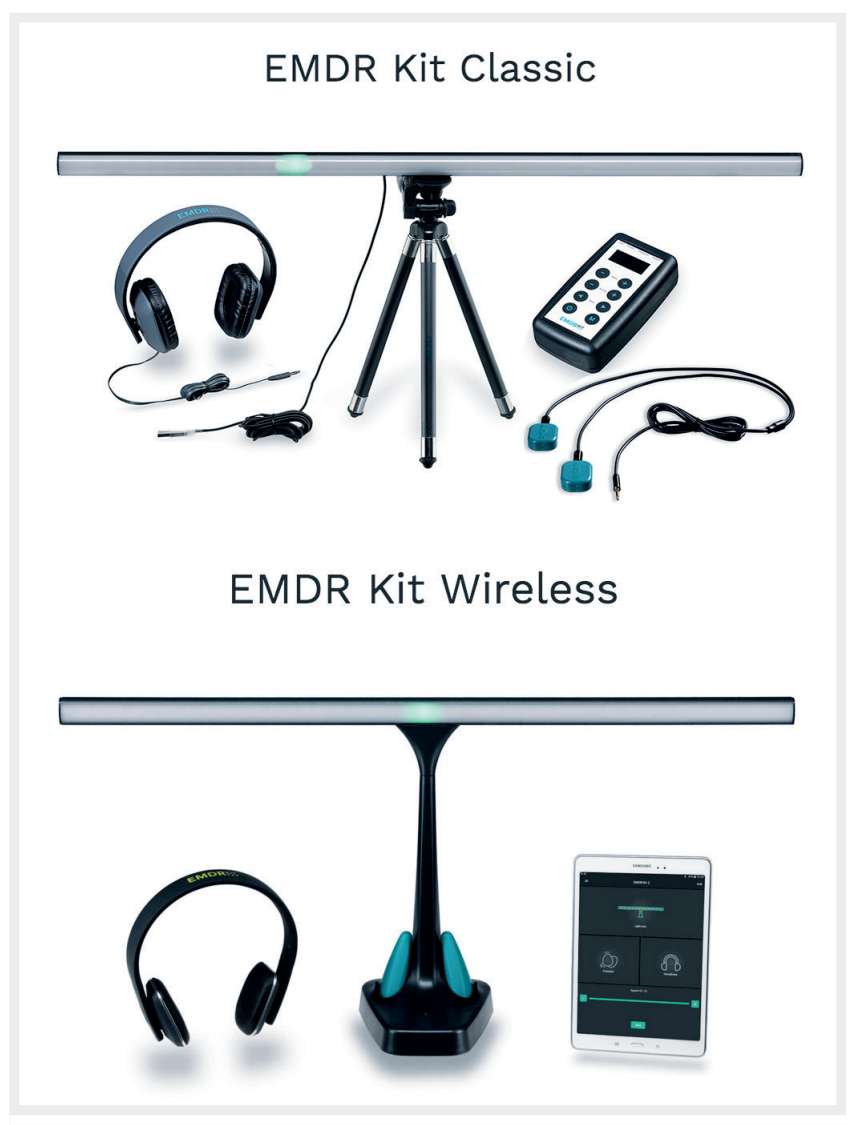

- Abb. 2 EMDR-Apparatur (oben herkömmlich, unten drahtlos), die im Netz für etwa 500 Dollar angeboten wird. Die abwechselnde Stimulation kann damit visuell (in unterschiedlichen Farben), akustisch (über Kopfhörer) und taktil (über die blaugrünen in beiden Händen zu haltenden Vibratoren) erfolgen. Quelle: nach https:// www.emdrkit.com/shop/ chanismus fand die Methode eine rasch steigende Zahl von Anhängern. Und obgleich Shapiro vor allem Patienten mit PTBS behandelt hatte, versuchten sich andere an Störungsbildern wie Depression sexueller Dysfunktion, Schizophrenie, Essstörungen und psychischem Stress bei Krebspatienten - mit sehr gemischten Ergebnissen, wie eine im Scientific American publizierte Übersicht berichtete [3].

Als Blinde behandelt wurden und ebenso gut wie Sehende ansprachen - ohne jegliche Augenbewegungen - wurden das „E“ und das „M“ in „EMDR“ angezweifelt. Und weitere Studien werden von den genannten Autoren wie folgt zusammengefasst: „Unter dem Strich lindert EMDR die Symptome traumatisch bedingter Angst besser als Nichtstun und möglicherweise auch besser als Gespräche mit einem aufmerksamen Zuhörer. Aber es gibt keinen Schnipsel von guter Evidenz dafür, dass EMDR den expositions-basierten Verfahren überlegen ist, die kognitive Verhaltenstherapeuten routinemäßig in den vergangenen Jahrzehnten angewandt haben “ [3].

Ein anderer Skeptiker schreibt: „Ranae Johnson gründetet das Rapid Eye Institut auf einer Heidelbeerfarm in Oregon, wo sie die Rapid Eye Technik lehrt. Diese tolle neue Therapie sei geeignet, 'um alte [Verhaltens-] Programme loszuwerden und zu löschen und damit den Weg zu mehr Bewusstheit für unsere Freude und unser Glück zu öffnen.' Es hilft uns, 'das Licht und die Spiritualität in uns zu finden, die schon immer da war.' Wie es scheint, bezahlen die Leute etwa 2000 Dollar für das Training und dafür, dass man so viele Heidelbeeren essen kann, wie man mag “ [9]. ${ }^{2}$ Schließlich zitieren die oben genannten Autoren [3] noch einen Satz des bekannten klinischen Psychologen der Harvard University Richard McNally, der dem EMDR-Verfahren gewissermaßen den Rest gibt: „Was bei der EMDR wirkt, ist nicht neu, und was neu ist, wirkt nicht.“3

Von ihren Befürwortern wird die EMDR als „ungewöhnliche Form der Psychotherapie“ bezeichnet [14], denn es wird während der Behandlung nicht nur geredet, sondern auch der Blick auf den sich recht rasch hin und her bewegenden Finger des Therapeuten gerichtet. Zunächst vergegenwärtigt sich der Patient gedanklich und emotional das traumatische Erlebnis und folgt dann bei ruhig gehaltenem Kopf mit den Augen den Handbewegungen des Therapeuten. Warum dadurch eine bessere Verarbeitung der belastenden Erlebnisse erfolgt und - so die Theorie - daher während der The-

2 Ranae Johnson has founded the Rapid Eye Institute on a blueberry farm in Oregon where she teaches Rapid Eye Technology. This amazing new therapy is used "to facilitate releasing and clearing of old programming, opening the way to awareness of our joy and happiness." It helps us "find light and spirituality within us that has always been there." Apparently, people are paying some $\$ 2000$ for the training and all the blueberries you can eat.

3 "What is effective in EMDR is not new, and what is new is not effective." Die Autoren bezeichnen diesen Satz als Paraphrasierung eines Ausspruchs des englischen Gelehrten und Schriftstellers Samuel Johnson, der über ein Manuskript gesagt haben soll: „Your manuscript is both good and original. But the part that is good is not original, and the part that is original is not good." - Ganz offensichtlich ein vernichtendes Urteil, weswegen das Zitat oft verwendet wird, obgleich es weder bei Samuel Johnson selbst und denen, die über ihn berichtet/geschrieben haben, zu finden ist (vgl. https://www.samueljohnson.com/goodorig. html). Dort findet man auch den Kommentar: „Simply put, as amusing as the quip is, it hasn't been found anywhere. Until someone can find a source for it, you're best off saying it's 'attributed, not found.' “ 
rapie eine stärkere Entlastung einsetzt, war bislang letztlich unbekannt. Man vermutete sowohl psychologische, physiologische als auch neurobiologische Wirkungsmechanismen der EMDR, wie beispielsweise eine erhöhte Kontrolle gegenüber gelernter Hilflosigkeit, einen Zusammenhang mit den Augenbewegungen im REMSchlaf (und dessen Bedeutung für Gedächtnis und Emotionsverarbeitung) oder eine bessere Integration der beiden Gehirnhälften [23]. Da die Bewegung der Augen nicht erforderlich ist, sondern auch durch abwechselnde Berührung oder Töne links und rechts ersetzt werden kann, blieb der Wirkungsmechanismus im Unklaren, zumal auch eine (im Hinblick auf den Therapeuten) verblindete Studie keinen Unterschied zwischen simultaner und alternierender bilateraler Stimulation finden konnte, wenngleich die Studie auch nochmals die Effektivität von EMDR bei Traumatisierung klar nachweisen konnte [33].

\section{Was ist PTBS?}

Die Arbeitsgemeinschaft der Wissenschaftlichen Medizinischen Fachgesellschaften e. V. (AWMF), der deutsche Dachverband von 178 Fachgesellschaften der Medizin, koordiniert seit 1995 die Entwicklung von medizinischen Leitlinien für Diagnostik und Therapie durch die einzelnen Wissenschaftlichen Medizinischen Fachgesellschaften. Gemäß ihrer S3-Leitlinie [4] auf die sich mehrere psychotherapeutische Fachgesellschaften geeinigt haben, wird unter einer PTBS das folgende verstanden: „[...] eine mögliche Folgereaktion eines oder mehrerer traumatischer Ereignisse (wie z. B. das Erleben von körperlicher und sexualisierter Gewalt, auch in der Kindheit (sexueller Missbrauch), Vergewaltigung, gewalttätige Angriffe auf die eigene Person, Entführung, Geiselnahme, Terroranschlag, Krieg, Kriegsgefangenschaft, politische Haft, Folterung, Gefangenschaft in einem Konzentrationslager, Natur- oder durch Menschen verursachte Katastrophen, Unfälle oder die Diagnose einer lebensbedrohlichen Krankheit), die an der eigenen Person, aber auch an fremden Personen erlebt werden können."

Nicht jedes Trauma führt zu einer PTBS, aber mit zunehmender Anzahl an erlebten Traumata steigt deren Wahrscheinlichkeit, wie Untersuchungen an Opfern von Krieg, Folter, politischer und sexueller Gewalt zeigten [13, 24, 25, 31, 36]. Solche durch andere Menschen verursachte Traumata („man made disaster“) wirken belastender und führen mit höherer Wahrscheinlichkeit zu einer PTBS als Unfälle oder Naturkatastrophen. Die Symptome treten innerhalb eines halben Jahres nach dem belastenden Erlebnis auf und dauern länger als einen Monat an.

\section{PTBS und EMDR im Mausmodell}

Wie gut, dass eine Arbeitsgruppe südkoreanischer Neurowissenschaftler (u. a. ein Physiker und ein Mediziner) sich der EMDR angenommen hat, nachdem sie einen Film darüber im Fernsehen gesehen hatten. ${ }^{4}$ Man verwendete hierzu zunächst das seit Jahr-

4

Diese Information verdanke ich meiner aus Südkorea stammenden Mit arbeiterin Dr. Eun-Jin Sim. Ich halte diesen Kontext der Entdeckung insofern für mitteilenswert, weil man sich bei der Lektüre, der in Nature publizierten Originalarbeit unwillkürlich fragt, wie ein physiologisches Labor auf die Idee kommt, eine solche Studie durchzuführen.

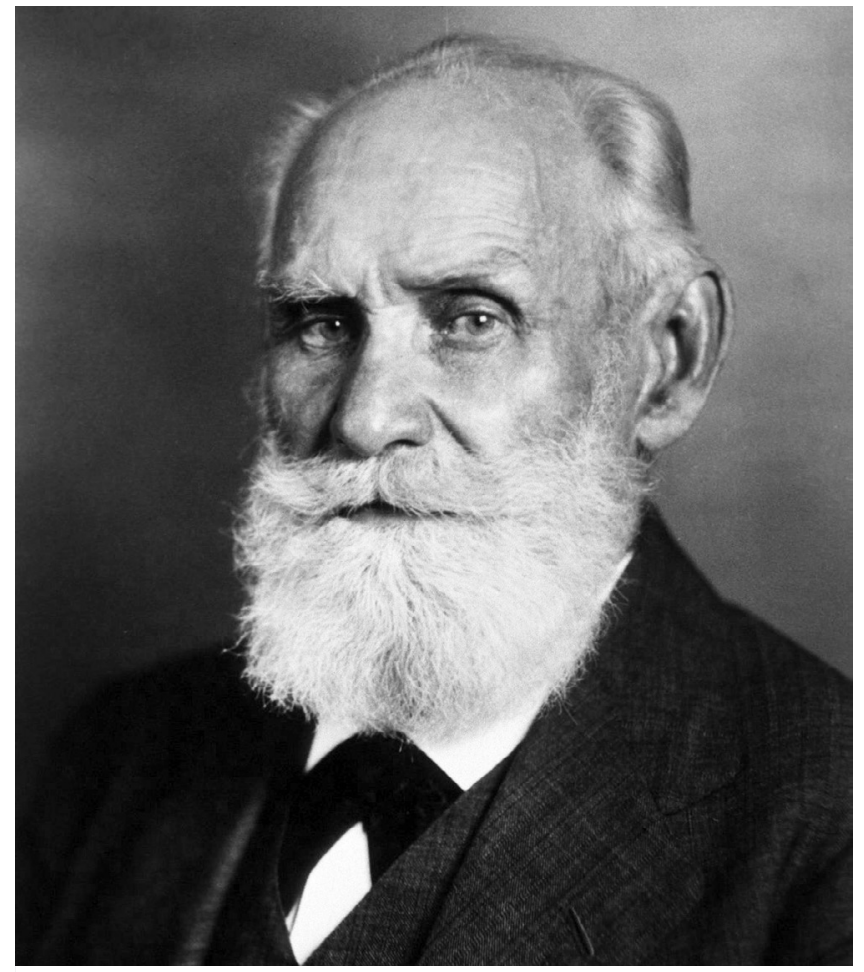

Abb. 3 Iwan Petrowitsch Pawlow (1849-1936), dessen Hunden die Spucke lief, wenn das Glöckchen klingelte. Diese durch das gemeinsame Darbieten des läutenden Glöckchens (CS) mit dem Anblick von Futter (US) gelernte Verbindung von Glöckchen mit Futter führt zum bedingen Reflex (CR) auf das Glöckchen mit vermehrtem Speichelfluss (bzw. Magensaftproduktion, die von Pawlow per Magensonde gemessen wurde). Quelle: (c) Wiki commons.

zehnten bekannte Tiermodell der PTBS ( $\bullet$ Abb. 1, links). In einem speziellen Käfig werden einer Maus ein schmerzhafter elektrischer Reiz über den leitenden „Fußboden“ des Käfigs appliziert (unkonditionierter Reiz) und zugleich über Lautsprecher ein Ton, also ein akustischer Reiz (konditionierter Reiz) dargeboten. Dadurch - man spricht von klassischer Konditionierung oder - nach ihrem Entdecker ( $\triangleright$ Abb. 3) - von Pawlowscher Konditionierung ${ }^{5}$ - werden die Reize miteinander assoziiert, so dass nach erfolgter Konditionierung der Ton zur gleichen Reaktion wie der schmerzhafte Elektroschock führt: Das Tier verfällt in eine Schockstarre (engl.: Freezing), was sich gut beobachten und quantifizieren lässt.

Auch Menschen verfallen zuweilen in eine solche Schockstarre und ein „Forme fruste“ derselben stellt beispielsweise der starre Blick in die Ferne dar ( $\$$ Abb.4), gleichsam durch das Gegenüber hindurch und in die Weite der Welt hinaus, ohne etwas Bestimmtes zu betrachten. Für Soldaten nach Kampfhandlungen ist dies schon lange unter dem Namen „Two Thousand Yard Stare“ bekannt ist und kann zu den Symptomen einer PTBS gehören.

Durch die in - Abb. 1 links gezeigte experimentelle Anordnung bringt man einem Versuchstier also die Reaktion der Schockstarre auf einen vollkommen harmlosen Ton bei, indem man den Ton

5 Dieses Paradigma geht bekannter Weise auf den russischen Psychologen Pawlow zurück, der damit die Lerntheorie begründete, die später vom US-amerikanischen Psychologen Burrhus Frederic Skinner (19041990) um das operante Konditionieren erweitert wurde. 


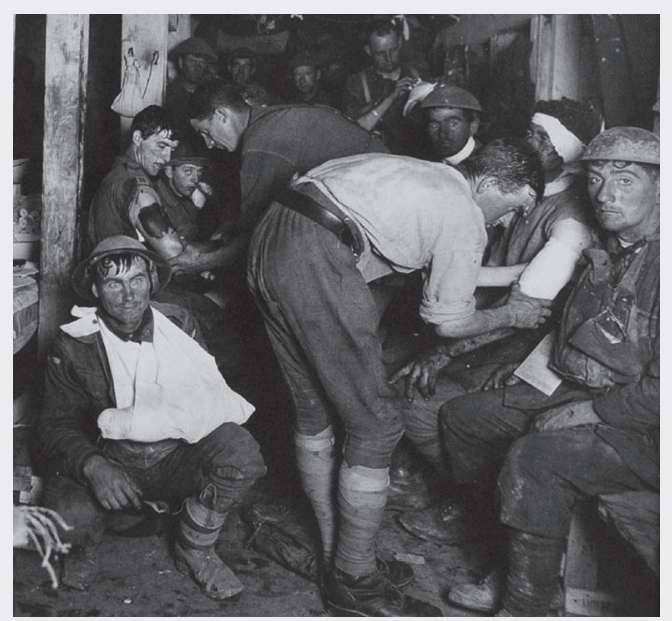

- Abb.4 Der starre Blick in die Ferne bei Soldaten nach traumatischen Kriegserlebnissen ist eine Form der Starre, die man auch beim Tier als Reaktion auf unkontrollierbare negative Erfahrungen beobachten kann. Das Bild wurde im Jahr 1917 während des ersten Weltkriegs in einem Feldlazarett für Verwundete aufgenommen und zeigt das Phänomen bei dem Soldaten mit der Armbinde links unten im Bild. Quelle: @ Wikipedia gemeinfrei

mit einem schmerzhaften elektrischen Schock kombiniert. Beim Menschen kommt es durch psychische Traumata zur posttraumatischen Belastungsreaktion. Bei ihr treten wiederkehrende, unfreiwillige und eindringliche belastende Erinnerungen auf, bei zugleich bestehenden Erinnerungslücken im Hinblick auf das Ereignis, Flashbacks und Albträume. Hinzu kommen körperliche Erregtheit (arousal) und wiederkehrende Angst, Gereiztheit, Schreckhaftigkeit, Konzentrations- und Schlafstörungen sowie Vermeidungsverhalten und ein negatives Selbstwertgefühl. Oft entwickelt sich zusätzlich eine Depression.

Das Auftreten Trauma-bedingter Ängste lässt sich nach diesem Modell gut erklären. ${ }^{6}$ Die Therapie besteht im Erlernen einer neuen Reaktion, die in der Unterdrückung der gelernten Reaktion besteht. Man spricht auch von Extinktionslernen (oder zu Deutsch „Löschung“) der Angstreaktion, obgleich es sich hier um einen Missnomer (wie man heute eine falsch gewählte Bezeichnung auf Neudeutsch nennt) handelt. Denn die gelernte Angstreaktion wird nicht gelöscht; es wird vielmehr eine zweite, die Angstreaktion hemmende Reaktion gelernt ${ }^{7}$, indem man den konditionierten Reiz (im Falle der Therapie in einer geschützten therapeutischen Situation) ohne den aversiven, unkonditionierten Reiz „aushalten lernt“. Hierbei hilft das Bewegen der Augen, wie in der EMDR schon

6 Ich selbst hatte das Erlebnis, von einem Kettenhund, der sich von der Kette losgerissen hatte, im Alter von 10 Jahren übel zugerichtet worden zu sein. Danach litt ich an einer klassischen Hundephobie und rannte in Todesangst davon, wenn auch nur der kleinste Pinscher in weiter Entfernung zu sehen oder zu hören war: Die Wahrnehmung eines Hundes hatte sich bei mir durch das Erlebnis, Opfer eines Raubtieres zu sein, fest mit größten Ängsten verbunden.

7 Vgl. hierzu die im Fachblatt Science publizierte sehr schöne Arbeit von Agren und Mitarbeitern [1] sowie die in der Nervenheilkunde kurz darauf erschienene Darstellung von deren Grundgedanken [35].

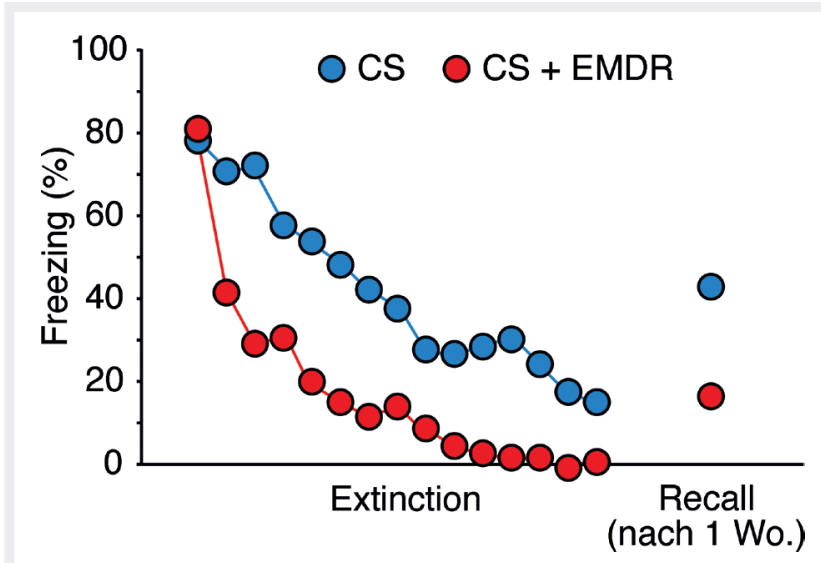

- Abb. 5 Vergleich des reinen Extinktionslernens (blau) mit dem von hin und her bewegtem LED-Licht begleitetem Extinktionslernen (rot). Der Unterschied ist mit $p<0,001$ hoch signifikant. Auf der $X$-Achse sind die einzelnen Durchgänge des Extinktionslernens und auf der Y-Achse die prozentuale Häufigkeit des Auftretens der Schockstarre (Freezing) bei den Tieren aufgetragen. Bei reinem Extinktionslernen reduziert sich das Freezing von $80 \%$ auf etwa $20 \%$, beim Extinktionslernen mit gleichzeitiger „EMDR“ erfolgt die Reduktion des Freezing rascher und geht auf praktisch Null zurück. Nach einer Woche wurde in gleicher Umgebung nur der CS (Ton) jedem Tier 3-mal dargeboten, um damit zu messen, wie gut die Extinktionsprozedur das Wiederauftreten der konditionierten Reaktion (Angst) auf den Ton (Recall) auch eine Woche nach der Prozedur zu unterdrücken vermag. Quelle: (modifiziert nach [5], S. 340, - Fig. 1b, Mitte; der Klarheit wegen wurden nur 2 der 4 Kurven aus der Originalabbildung reproduziert).

seit etwa zwei Jahrzehnten behauptet wird und jetzt im Mausmodell gezeigt werden konnte.

Das Verfahren, wie man EMDR bei Mäusen durchführen kann, ist schematisch in - Abb. 1 rechts dargestellt: Das Tier im zylindrischen Käfig ist rundherum von LEDs umgeben. Wird nach der Konditionierung nun erneut der Ton (CS) präsentiert und zugleich durch eine hin- und herwandernde Beleuchtung der Blick bzw. die Aufmerksamkeit des Tieres hin- und hergelenkt, dann lässt sich experimentell die Frage beantworten, ob ein solches bewegtes Licht zu einem besseren Verlernen der Angstreaktion führt, bzw. zu einem besseren Neulernen der Reaktion, keine Angst zu haben. Dies war tatsächlich der Fall, wie $>$ Abb. 5 zeigt. Die Angstreaktion der Versuchstiere (Freezing) nimmt rascher ab und erreicht ein deutlich geringeres Niveau unter „Extinktion bei gleichzeitiger „EMDR“ als unter alleiniger „Extinktion“. Um sicher zu sein, dass es an der bewegten Stimulation liegt und ebenso an der Gleichzeitigkeit dieser Stimulation mit dem Ton, wurden drei Kontrollbedingungen verwendet,

- die Extinktion bei während der Präsentation des Tons angeschalteten LEDs,

- die Extinktion bei während der Präsentation des Tons synchron blinkenden LEDs und

- die Extinktion bei im Wechsel mit der Präsentation des Tons hin-und her bewegten LEDs, also keiner gleichzeitigen Darbietung von Ton und „laufenden“ LED-Leuchten. 
Unter diesen 3 Kontrollbedingungen war die Extinktion nicht besser als bei alleiniger Extinktion ohne LEDs. Daraus muss man schließen, dass es die gleichzeitig zur Darbietung des Tones „laufenden“ LEDs sind, die den Effekt bewirken.

Es gab zwar seit Jahren methodisch sehr gut gemachte klinische Studien zur EMDR [33], aber irgendetwas von Zauberei hatte die Prozedur dennoch aus meiner Sicht über all die Jahre. Allein aus diesem Grund ist das in \ Abb. $\mathbf{5}$ dargestellte Ergebnis im Grunde schon eine kleine Sensation: EMDR lässt sich bei Mäusen durchführen und hat in diesem Modell einen deutlich stärkeren Effekt als alleinige Extinktion! Wer also dazu neigte, EMDR eher für Voodoo zu halten, wird durch dieses Ergebnis nun eines Besseren belehrt. Im Mausmodell jedenfalls hat die Methode genau den Effekt, der von den Praktikern und Befürwortern der EMDR seit etwa zwei Jahrzehnten beim Menschen behauptet bzw. - wie man nun eher geneigt ist zu sagen - beschrieben wurde. Damit gaben sich die Autoren jedoch keineswegs zufrieden. Im Gegenteil: Die Arbeit ging damit überhaupt erst so richtig los! Denn dafür gibt es in der Medizin ja Tiermodelle: Am Modell kann man experimentelle Untersuchungen durchführen, die am Menschen entweder nicht möglich sind oder sich aus ethischen Gründen verbieten.

„Der Colliculus superior ist eine der am besten untersuchten Strukturen des Gehirns, und mit jedem neuen Artikel [über ihn] scheint die Komplexität der ihm zugeschriebene Funktion bei der Verhaltenskontrolle komplexer zu werden. “ Mit diesem Satz beginnen Basso und May [6] bezeichnenderweise ihren Review des Colliculus superior $^{8}$, einer histologisch mehrschichtigen, makroanatomisch identifizierbaren Struktur des Mittelhirns ( $\triangleright$ Abb. 6). $\mathrm{Er}$ ist zum Sehen im Sinne von Objekterkennung nicht erforderlich, wie u. a. Läsionsstudien an Katzen zeigten. In diesen Studien zeigte sich aber auch, dass ohne ihn die Orientierung auf ein Objekt nicht mehr funktioniert. Seit den 1970er-Jahren wurde er mit Augenbewegungen in Verbindung gebracht, seit den 90er Jahren zunehmend aber auch mit Aufmerksamkeitsprozessen - mit oder ohne begleitende motorische Aktivität („overt“ und „covert“ attention shifts) [26]. Man spricht von „spatial indexing“ [22] und mittlerweile wird ihm die Funktion einer Salienzkartierung zugeschrieben[38], die gleich zweifach vorliegt - erstens als Karte bedeutsamer Stimuluseigenschaften („saliency map“; oberflächliche Schichten) und zweitens als Karte der Handlungspriorisierung („priority map“; tiefere Schichten).

Aufgrund seiner Funktionen im Hinblick auf Salienzkodierung, Aufmerksamkeit und Augenbewegungen lag es nahe, durch Einzelzellableitungen aus den tieferen Schichten des Colliculus superior den Mechanismen der durch EMDR beschleunigten Verminderung der konditionierten Angstreaktion nachzugehen. Man präsentierte hierzu zunächst in randomisierter Reihenfolge bei Mäusen die in den Konditionierungs- und Extinktionsexperimenten verwendeten Stimuli, also den Ton oder die drei visuellen Reize (LEDs dauernd an, LEDs blinken, LED-Licht wandert hin und her) jeweils für 30 Sekunden. Hierbei wurden die meisten Neuronen durch den hin- und herwandernden visuellen Reiz (36,1\%) aktiviert (Ton: 13,8\%, Dauerlicht: 21,1\%, Blinklicht: 28,4\%; vgl. [5], Extended Data • Fig. 2).

8 Obwohl die Struktur paarig angelegt ist, spricht man in der Literatur oft von „dem“ Colliculus superior, meint aber damit immer beide Colliculi superiores.

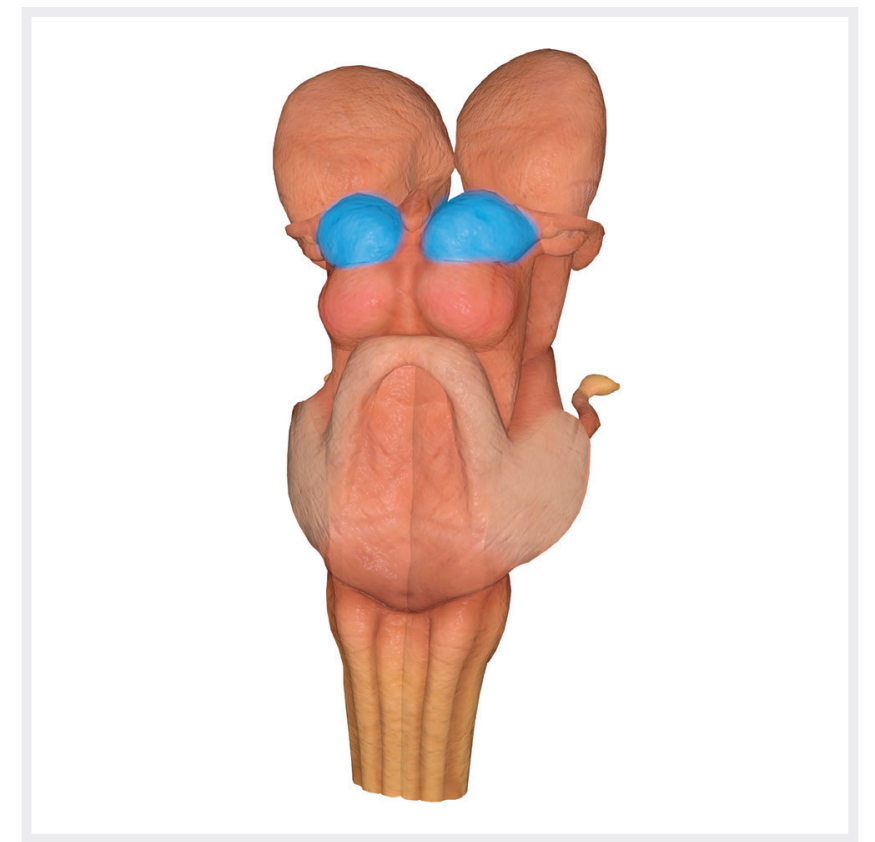

- Abb. 6 Die beiden oberen Hügelchen (in der Abbildung blau) sind mit den beiden unteren Hügelchen Teil der Vierhügelplatte, die ihrerseits zum Mittelhirn gehört. Während die unteren Hügel Teil der Hörbahn sind, gehören die oberen Hügel zum Sehsystem. Während sie bei Säugetieren eher klein ausgebildet sind, erhalten sie bei Fischen, Fröschen und vor allem Vögeln den Hauptteil der Fasern von der Retina als Input und bilden bei den genannten Tieren damit im Wesentlichen deren Sehsystem. Da man mit diesen „niederen“ Tieren leichter experimentieren konnte, wurde bis Ende der 1960er-Jahre angenommen, dass die Funktion der oberen Hügel vor allem das Sehen selbst darstellt. Dies änderte sich in den 1970er- und 1980er-Jahren, nachdem Experimente an Affen gezeigt hatten, dass die beiden oberen Hügel vor allem für Augenbewegungen zuständig sind. Als Fortschritte der experimentellen Methodik die Untersuchung von sich bewegenden Tieren (in den frühen Experimenten war der Kopf befestigt, um die neurophysiologischen Ableitungen „in Ruhe“ durchführen zu können) erlaubten, wurde im Verlauf der letzten 2 Jahrzehnte immer klarer, dass der Colliculus superior auch für Kopf- und Armbewegungen zuständig sein kann und eher so etwas wie ein Zentrum der Kontrolle der Aufmerksamkeit darstellt. Er erhält dazu seinen Input nicht nur (in den oberflächlichen Schichten) von den Augen, sondern auch von den Ohren (über den Colliculus inferior) und vor allem vom Kortex zur Top-Down-Kontrolle der Aufmerksamkeit (tiefere Schichten zur Repräsentation der Priorisierung beim Handeln) (Quelle: Thieme Verlag).

Aufgrund dieses Befundes entschloss man sich, entsprechende Ableitungen auch bei der Extinktion von Angstverhalten vorzunehmen, wozu man entweder nur den Ton (reine Extinktion) oder den Ton und den bewegten visuellen Reiz darbot (Extinktion plus EMDR). Hierbei zeigte sich eine Aktivierung von mehr Neuronen (63,3\%) bei der Bedingung „Extinktion plus EMDR“ im Vergleich zur Bedingung „Extinktion“ (33,7\%). Die Größe der positiven neuronalen Antwort jeder einzelnen Maus war mit deren Angstverhalten („Freezing“) beim Test eine Woche später („retention“) signifikant korreliert $(r \sim 0,5)$. Bei manchen Neuronen kam es unter der Stimulation auch zu einer Hemmung der Aktivität die jedoch unter beiden Bedingungen („Extinktion“: 8,2\%; „Extinktion plus EMDR“: 10,2\%; vgl.[5], \ Fig. 1e) erstens nur einen geringen An- 


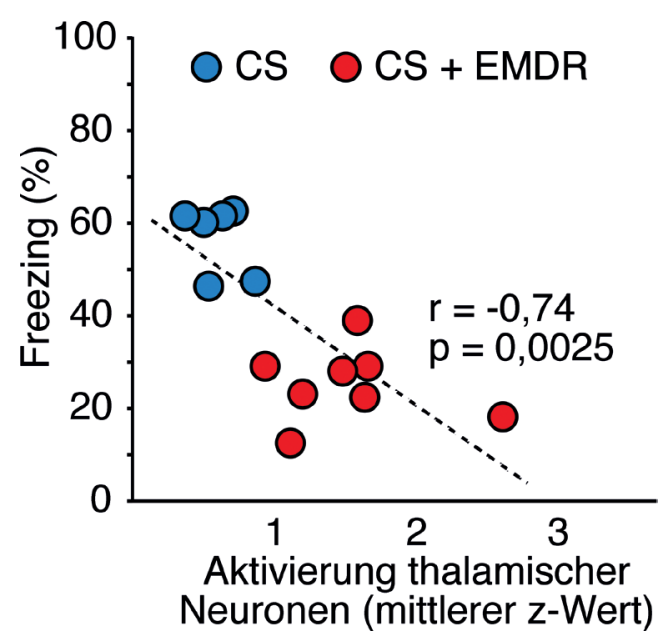

- Abb.7 Freezing-Verhalten in Abhängigkeit von den elektrischen Antworten der positiv auf den Stimulus reagierenden Neuronen im mediodorsalen Thalamus (auf der X-Achse nach rechts abgetragen: mittlere z-Werte der aktivierten Neuronen der 6 Mäuse in der Bedingung „Extinktion“ und der 8 Mäuse in der Bedingung „Extinktion+EMDR“. Quelle: [5], > Fig. 2e).

teil der Neuronen betraf und zweitens nicht mit dem Freezing eine Woche nach der Extinktion korreliert war (vgl. [5], Extended Data - Fig.3). Daraus leiteten die Autoren ab, dass die Wirkung der EMDR-Prozedur im Mausmodell über eine verstärkte Aktivierung des Colliculus superior vermittelt wird.

Diese Aktivierungsverstärkung wird dem mediodorsalen Thalamus übermittelt, der Inputfasern aus dem Colliculus superior erhält und seinerseits sowohl in den präfrontalen Kortex als auch in den Mandelkern (Amygdala) projiziert. Im Thalamus fand man zunächst keine Abhängigkeit der Anzahl der aktivierten Neuronen von der Extinktions-Bedingung, d. h. ohne (35,8\% positive response) oder mit EMDR (34,05 positive response; vgl.[5], - Fig. 2b) war die Anzahl aktivierter Neuronen gleich. Die elektrischen Antworten der positiv auf den Stimulus reagierenden Neuronen waren jedoch unter der Bedingung „Extinktion plus EMDR“ hoch signifikant stärker als unter der Bedingung „Extinktion“. Zudem waren sie signifikant ( $p=0,0025)$ mit einer Verminderung des Freezing korreliert $(r=-0,74 ;>$ Abb. 7). Keine solchen Zusammenhänge gab es bei den Neuronen, die mit einer verminderten Aktivierung reagierten.

Mittels optogenetischer Verfahren konnte weiterhin gezeigt werden, dass bei einem Abschalten („silencing“) der Verbindung vom Colliculus superior zum mediodorsalen Thalamus während des Extinktionslernens mit EMDR dieses nicht mehr funktioniert. Umgekehrt konnte man mit einer Laser-Stimulation von Neuronen des Colliculus superior während des Extinktionslernens den Effekt der EMDR erzeugen. Auf diese Weise ließ sich die Kausalität der gefundenen Verbindung vom Colliculus superior zum mediodorsalen Thalamus für den Effekt der EMDR auf das Extinktionslernen klar nachweisen (die Autoren sprechen von „notwendiger und hinreichender Bedingung“, [5], S. 340).

Wie aber macht der Thalamus das „Freezing“, d. h. über welche seiner Projektionen wirkt sich dieses Signal weiter aus? Man wusste bereits, dass die basolaterale Amygdala zwei Neuronenpopu- lationen enthält, die entweder die konditionierte Angstreaktion oder die Extinktion kodieren [16] und Projektionen zu unterschiedlichen Neuronenpopulationen des präfrontalen Kortex senden [32].

Die von Baek und Mitarbeitern durchgeführten Einzelzellableitungen in der basolateralen Amygdala zeigten bei den die Angstreaktion kodierenden Neuronen („Fear cells“) eine verminderte Aktivierung während der Extinktionsdurchgänge mit EMDR im Vergleich zu Extinktionsdurchgängen ohne EMDR ( $>$ Abb. 8). Für die Aktivierung der die Extinktion kodierenden Neuronen („Extinction cells“) gab es dagegen keinen Unterschied zwischen beiden Extinktionsbedingungen. Entsprechend zeigte sich eine hochsignifikante Korrelation der Aktivierung der Fear cells mit dem Freezing ( $r=0,63 ; p=0,007$; Abb. 9) und keine signifikante Korrelation der Aktivierung der Extinction cells mit dem Freezing.

Wiederum mittels optogenetischer Methoden wurden in einem weiteren Experiment die Projektionen vom mediodorsalen Thalamus zur basolateralen Amygdala während der Extinktion ausgeschaltet („silencing“). Dies führte zu einem kompletten Verlust des Effekts der EMDR auf die Extinktion. Wie aber genau führt der erregende Input vom Thalamus in der Amygdala zu einer geringeren Aktivierung?

Erneut wurden optogenetische Stimulationsexperimente des Thalamus mit Ableitungen von der Amygdala durchgeführt, wo sich etwa 5 ms nach der thalamischen Stimulation exzitatorische Potentiale, und etwa 10 ms nach der thalamischen Stimulation inhibitorische Potentiale, zeigten ( $\triangleright$ Abb. 10).

Die in diesen Ableitungen gefundenen zeitlichen Latenzen sind mit einer monosynaptischen Erregung und einer bisynaptischen Hemmung von Neuronen im Mandelkern durch den Thalamus vereinbar, d. h. mit einem Modell, das sowohl (monosynaptische) erregende als auch mittels eines hemmenden Interneurons bewirkte hemmende Verbindungen beinhaltet ( $\mathbf{A} \mathbf{b b} \mathbf{b} \mathbf{1 1})$.

Bei einer durch EMDR verstärkten Extinktion kommt es zu vermehrter Hemmung der Amygdala und verminderter Erregung, d. h. zu einem erhöhten Verhältnis von Hemmung zu Erregung und damit zu einem verminderten Output von der Amygdala zu weiteren Angst-generierenden Regionen. Durch diesen Mechanismus wird die Wirkungsweise von EMDR im Sinne einer verstärkten Extinktion von traumatisch entstandener Angst plausibel.

\section{Diskussion: Normalisierung im Gehirn}

Nach den hier diskutierten Erkenntnissen - Extinktion mit EMDR wirkt rascher und stärker als Extinktion allein durch Re-Exposition - wundert es nicht, dass die EMDR in neueren und neuesten Reviews und Metaanalysen zur klinischen Wirksamkeit - nicht zuletzt im Vergleich zur kognitiven Verhaltenstherapie (cognitive behavioral therapy, CBT) - als wirksamer eingestuft wird [11, 19]. Auch erscheint die Rate der Therapie-Abbrecher geringer, was ebenfalls durch den rascheren Wirkungseintritt und die stärkere Wirkung erklärt werden kann [40]. Vor allem die Symptome der Intrusionen und der Erregung scheinen besser auf EMDR anzusprechen, nicht hingegen die Depression. Auch dies würde dazu passen, das der Colliculs superior Salienz - bis hin zu unmittelbarer Bedrohung - kodiert und in Reaktionen umsetzt, die den Organismus bei lebensgefährlicher Bedrohung in Sicherheit bringen [12]. 


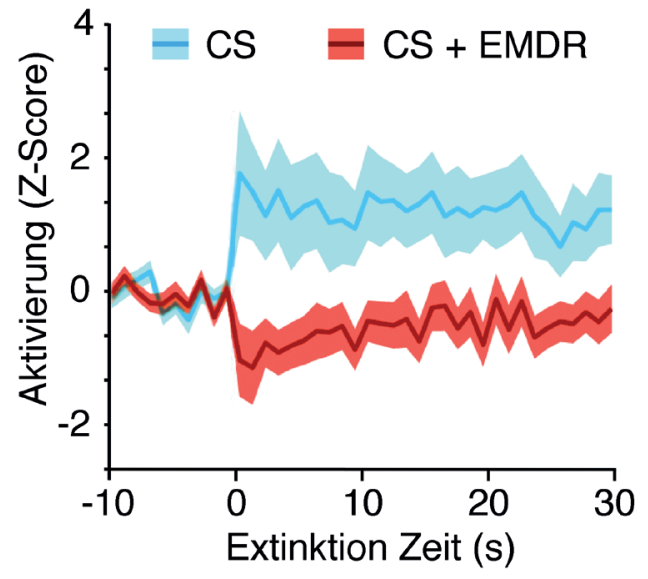

Abb. 8 Durchschnittliche Aktivierung (Mittelwerte: dunkel, und Standardabweichungen: hell) der Fear cells in der basolateralen Amygdala während Extinktionsdurchgängen ohne (CS; blau; 34 Neuronen) und mit gleichzeitiger EMDR (rot; 42 Neuronen). Der Unterschied ist mit $p=0,0065$ hoch signifikant. Quelle: nach [5], S. 341, D Fig. 3c.

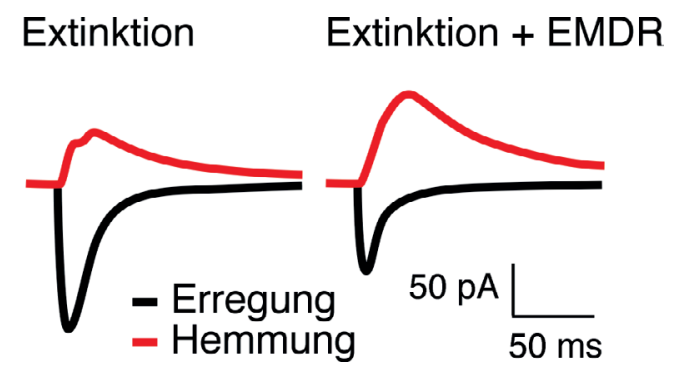

- Abb. 10 Schematisiert dargestellte Beispiele von abgeleiteten exzitatorischen (schwarz) und inhibitorischen (rot) Potenzialen bei Extinktion (links) bzw. Extinktion mit EMDR (rechts), die durch optogenetische Stimulation der Verbindungsfasern vom Thalamus zur Amygdala ausgelöst wurden Typ. Quelle: nach [5], S. 342, > Fig. 4e; der Maßstab für die Stomstärke (picoAmpere) wurde angeglichen.

Wie aber kommt die dämpfende Wirkung rascher Augenbewegungen auf Salienz-Output-Signale im Colliculus superior überhaupt zustande? Hierzu sagen die Autoren in ihrer Diskussion nichts. Jüngst publizierte Erkenntnisse zu Informationsverarbeitungsleistungen des Colliculus superior lassen jedoch zumindest einige Vermutungen zu. Zunächst legen experimentelle Daten zur Kontrolle von Sakkaden nahe, dass er die mathematische Funktion der Normierung bzw. Normalisierung ${ }^{9}$ leistet [40].

9 Im Deutschen werden die beiden Wörter - „Normierung“ und „Normalisierung“ - für den gleichen Sachverhalt verwendet. Da man im Englischen von „normalization“ spricht und die Literatur zur „Computational Neuroscience“ (dieser Ausdruck hat keine deutsche Übersetzung!) vor allem in englischer Sprache abgefasst ist, verwende ich im Folgenden das Wort „Normalisierung“.

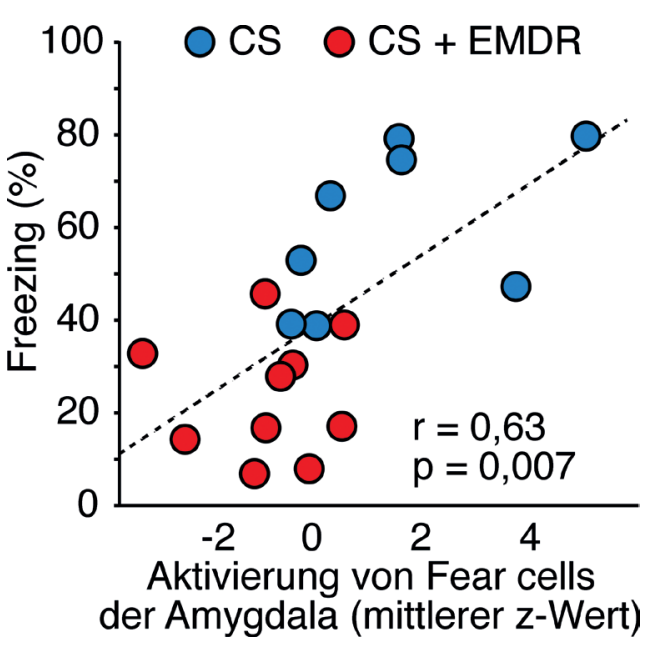

- Abb.9 Korrelation der Häufigkeit des Auftretens von Angstverhalten (\% Freezing) mit der mittleren Aktivierung der Fear cells in der Amygdala während der Extinktion bei den Tieren mit alleinigem Extinktionslernen ( $n=8$, blaue Punkte) und Tieren mit Extinktionslernen und gleichzeitiger EMDR ( $n=9$; rote Punkte). Quelle: nach [5], S. 341, > Fig. 3e.

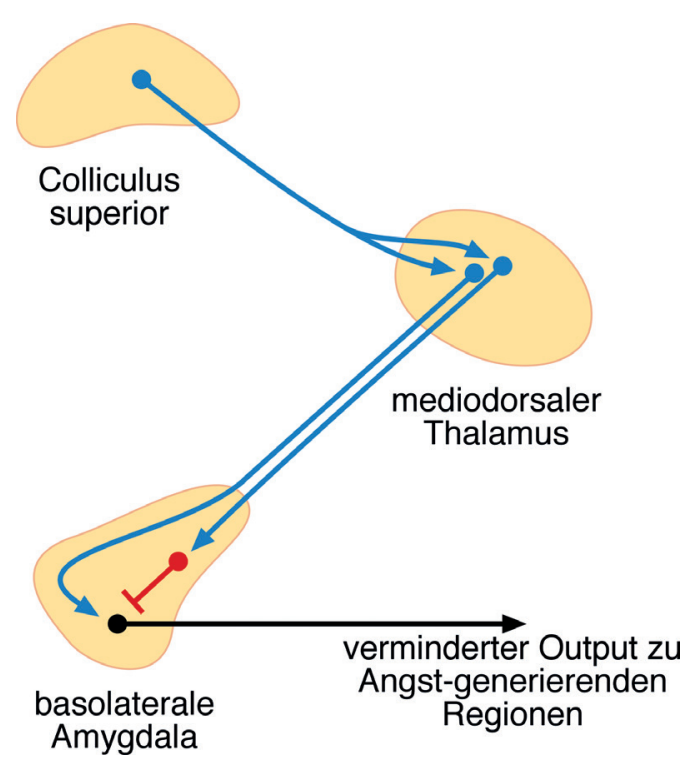

- Abb. 11 Modell der Verschaltung von Colliculus superior, mediodorsalem Thalamus und basolateraler Amygdala beim Verlernen von konditionierter Angst. Eine EMDR-begleitete Extinktion führt zu einer vermehrten Aktivierung des Colliculus superior, die über den Thalamus die Amygdala erreicht. Dort erhöht sich das Verhältnis von inhibitorischen zu exzitatorischen Potenzialen. Quelle: modifiziert nach [17], S. 335, > Fig. 1c.

In der Medizin meint man mit „Normalisierung“ meist, dass sich ein krankhafter Zustand (ein Messwert liegt entfernt von der gesunden Norm; z. B. „Fieber“) wieder dem Normwert $\left(37^{\circ} \mathrm{C}\right)$ annähert. In der Mathematik bedeute Normalisierung jedoch etwas ganz anderes, nämlich die Skalierung des Wertebereichs einer Variablen auf 
einen bestimmten Bereich, üblicherweise zwischen 0 und 1 (bzw. $100 \%)$. In der linearen Algebra normiert man einen Vektor, indem man seine Länge auf 1 verkürzt oder verlängert, die Richtung jedoch beibehält. Man nennt ihn dann Einheitsvektor. Viele numerische Rechenverfahren sind darauf angewiesen, dass der Wertebereich beim wiederholten Anwenden einer Funktion weder zu groß noch zu klein wird, da der Algorithmus sonst nicht mehr funktioniert. - Unser Gehirn auch!

Eine Reihe grundlegender Funktionen des Gehirns beruhen auf der mathematischen Operation der Normalisierung. Unsere Sinne sind beispielsweise in der Lage, auf Reize differenziell zu reagieren, obwohl diese über viele Größenordnungen hinweg variieren. ${ }^{10}$ Auch der Kortex reagiert auf starken Input stärker als auf schwachen, seine Aktivität ist aber nie „am Anschlag“, d. h. es kommt nie zur Sättigung wie beispielsweise bei einem überbelichteten Bild.

Beim Lernen, d. h. bei der Zunahme von Synapsenstärken durch die Verarbeitung von Input im Sinne der Neuroplastizität, darf es ebenfalls langfristig nie zu einer Zunahme der Stärke aller Synapsen und damit der postsynaptischen Gesamtaktivität des Systems kommen, weil sonst jeder Lernprozess letztlich zu einem epileptischen Anfall führen würde. Bei der Simulation neuronaler Netzwerke müssen daher die Synapsengewichtsvektoren normalisiert, d. h. auf die Länge 1 gebracht, werden.

Über lange Zeit war die Frage, ob und wie die mathematische Funktion der Normalisierung im Gehirn bewerkstelligt wird, unbeantwortet. Mittlerweile wurde experimentell gefunden, dass Normalisierung im Kortex sowohl bei der Informationsverarbeitung [28] als auch beim Lernen [37] vorkommt [34].

Auch im Colliculus superior findet Normalisierung statt [6]. Dass er nicht nur für Sakkaden zuständig ist, und seine Input- und Top-down-getriebenen Aufmerksamkeitskarten auch Aufmerksamkeitsprozesse unabhängig von Augenbewegungen steuern, wurde oben diskutiert. Modelle der Aufmerksamkeit als Implementierung der Normalisierungsfunktion gibt es ebenfalls bereits [30]. Studien zur attention bias variability, einem Maß für die Fluktuation der Aufmerksamkeit hin zu und weg von Trauma-assoziierten visuellen Stimuli zeigen eine deutlich höhere Variabilität bei PTBS [27] und einen Zusammenhang der Variabilität mit der Symptomstärke sowie der Symptomreduktion (und attention bias variability reduction) nach attention control training [42]. Dies ist mit einer - mathematisch gemeinten - Normalisierung durchaus vereinbar.

Im Umkehrschluss ließe sich vermuten, dass bei PTBS die Normalisierungsfunktion des Colliculus superior gestört ist - entweder durch „verzerrte“ (d. h. an bestimmten Stellen überaktive) Karten oder durch überrepräsentierten Trauma-spezifischen (dysfunktionalen) Top-down Input. Man braucht wenig Phantasie, um sich die Symptomatik innerhalb eines solchen Bezugsrahmens vorzustellen. Es würde bedeuten, dass letztlich ein verminderter Output der dysfunktionalen Prioritätenkarte über den Thalamus und das hemmende Interneuron in der Amygdala diese enthemmt. Unmittelbare Kontrolle der Aufmerksamkeit durch visuelle Blickfolgebewegungen könnte über die im Colliculus superior gleichsam eingebaute Normierungsfunktion die Normalisierung der Aktivität bewirken und traumatisch verursachte Verzerrungen in der

10 Das seit Weber und Fechner bekannte Logarithmieren von Sinnesdaten löst dieses Problem des dynamischen Bereichs nicht vollständig.
Prioritätenkarte mindern. Die hierfür nötigen Verbindungen zwischen der Input-Salienzkarte und der (verhaltensrelevanten) Output-Prioritätenkarte sind Teil der bekannten internen Verschaltungen der Struktur, d. h. - hard wired - vorhanden. Da die Normalisierung zudem auch für kontralaterale visuelle Felder nachgewiesen wurde, könnte dies die Wirksamkeit der seitenalternierenden Stimulation (im Gegensatz zu vorne-hinten oder oben-unten) erklären. Zusammenfassend könnte man damit sagen, dass sich unter EMDR, oder genauer unter alternierender bilateraler Stimulation (ABS) die Normalisierung schneller normalisiert. Dadurch erhalten dann die downstream nachfolgenden Strukturen (Thalamus, Amygdala) wieder geregelteren, weniger stark pathologisch verzerrten Input. Insofern ist alternierende bilaterale Stimulation ein spezifischer Reiz, der das Umlernen der konditionierten Angstreaktion begünstigt, weil er nur in dieser Form den von der Natur vorgegebenen Mechanismus in den tieferen Schichten des Colliculus superior anzusprechen vermag und daher anderen unspezifischen Stimulationsarten überlegen ist.

Interessant ist hierbei noch der Gesichtspunkt, dass der Colliculus inferior bekanntermaßen den Time-Code beherrscht, d. h. Aktionspotentiale in ihm nicht nur durch ihre Feuerrate (Rate-Code) Informationen kodieren, sondern durch den (auf Microsekunden) genauen Zeitpunkt ihres Auftreten. Dieser Code enthält dadurch um 2-3 Größenordnungen mehr Information pro Aktionspotential. Gefunden wurde dies durch Untersuchungen über das Richtungshören bei der Schleiereule [20], bei dem eine Raumkarte aus Laufzeitdifferenzen zwischen am rechten und linken Ohr eintretendem Schall berechnet wird. ${ }^{11}$ Diese Karte wird mit den Raumkarten im Colliculus superior abgeglichen, die wahrscheinlich den gleichen Code verwenden. Nicht umsonst verwenden die Vögel den Colliculus superior zum Sehen, denn sie brauchen eine schnelle visuelle Informationsverarbeitung beim Navigieren in der Luft. ${ }^{12}$ Mit Rate-code ist das niemals zu schaffen. Andererseits muss ein Time-code zur Produktion von Verhalten immer in Muskelaktionen umgesetzt werden, die über einen Ratenkode funktioniert: hohe Feuerrate an der muskulären Endplatte bewirkt stärkere Muskelkontraktion. Wo und wie genau diese Umsetzung des einen Kodes in den anderen erfolgt, konnte bis heute nicht vollständig geklärt werden, der Colliculus superior ist jedoch der heißeste Kandidat hierfür [20, 29, 38, 39]. Die Therapie mit ABS würde aus dieser Sicht einen sehr wichtigen grundlegenden Mechanismus der Gehirnfunktion ausnutzen.

Es wird sich zeigen, ob die Methoden der kognitive Neurowissenschaft und insbesondere der funktionellen Bildgebung in der Lage sein werden, derartige Hypothesen auch im Menschen in-vivo zu testen. EMDR im MRT geht - auch und gerade in Ulm [15]. Über

11 Bei einer Schallgeschwindigkeit von 300 m/s, entsprechend $30 \mathrm{~cm} /$ ms, ist der Unterschied des Eintreffens von Schall am linken und rechten Ohr der Eule (Entfernung ca. 6 cm) maximal 200 Mikrosekunden, beim frontalen Eintreffen des Schalls unter verschiedenen wenigen Winkelgraden geht es nur noch um wenige Mikrosekunden Differenz, die einen Informationsgehalt haben müssen, weil die Eule sonst beim nächtlichen Jagen ihre Beute (Maus) nicht finden würde.

12 Wer dies nicht glaubt, beobachte einmal einen Schwarm Schwalben beim Abendessen (von Fluginsekten) - ein unglaubliches Gewirr von Individuen, die abenteuerliche Flugmanöver ausführen und dabei nie kollidieren. 
die klinische - diagnostische und therapeutische - Fruchtbarkeit der hier vorgestellten neuen Erkenntnisse kann man gespannt sein.

\section{Danksagung}

Ich danke meinen Mitarbeitern Georg Grön, Thomas Kammer, Eu-Jin Sim und Visal Tumani für unsere Diskussionen zum Thema.

\section{Literatur}

[1] Agren T et al. Disruption of reconsolidation erases a fear memory trace in the human amygdala. Science 2012; 2337: 1550-1552.

[2] Angelidis A et al. I'm going to fail! Acute cognitive performance anxiety increases threat-interference and impairs WM performance. PLoS ONE 2019; 14: e0210824.

[3] Arkowitz H, Lilienfeld SO. EMDR: Taking a Closer Look. Can moving your eyes back and forth help to ease anxiety? Scientific American 1.8.2012 (https://www.scientificamerican.com/article/emdr-taking-acloser-look/; abgerufen am 4.3.2019)

[4] AWMF. S3 - Leitlinie: Posttraumatische Belastungsstörung, AWMF-Registernummer 051/010 (offline, Archiv: Stand 08/2011)

[5] Baek J et al. Neural circuits underlying a psychotherapeutic regimen for fear disorders. Nature 2019; 566: 339-343.

[6] Basso MA, May PJ. Circuits for action and cognition: A view from the superior colliculus. Annu Rev Vis Sci 2017; 3: 197-226.

[7] Beltramo R, Scanziani M. A collicular visual cortex: Neocortical space for an ancient midbrain visual structure. Science 2019; 363: 64-69.

[8] Bishop SJ. Neural mechanisms underlying selective attention to threat. Ann N Y Acad Sci 2008; 1129: 141-152.

[9] Carroll RT. The Sceptic's Dictionary: eye movement desensitization and reprocessing 2019 (EMDR). (http://skepdic.com/emdr.html; abgerufen am 4.3.2019)

[10] Chen L, Zhang G, Hu M, Liang X. Eye movement desensitization and reprocessing versus cognitive-behavioral therapy for adult posttraumatic stress disorder: systematic review and meta-analysis. J Nerv Ment Dis 2015; 203: 443-451.

[11] Chen $R$ et al. The efficacy of eye movement desensitization and reprocessing in children and adults who have experienced complex childhood trauma. Front Psychol 2018; 9: 534.

[12] Evans DA et al. A synaptic threshold mechanism for computing escape decisions. Nature 2018; 558: 590-594.

[13] Gaviria SL et al. Socio-demographic patterns of posttraumatic stress disorder in Medellin, Colombia and the context of lifetime trauma exposure, Disaster Health 2016; 3: 139-150.

[14] Hase M, Leutner S, Tumani V, Hofmann A. Eye movement desensitization and reprocessing (EMDR). Eine ungewöhnliche Form der Psychotherapie. Deutsches Ärzteblatt 2013; 12(11): 512-514.

[15] Herkt D et al. Facilitating access to emotions: Neural signature of EMDR stimulation. PLoS ONE 2014; 9(8): e106350.

[16] Herry $C$ et al. Switching on and off fear by distinct neuronal circuits. Nature 2008; 454: 600-606.

[17] Holmes A. Brains that learn not to fear. Nature 2019; 566: 335-336.

[18] lacoviello BM et al. Attention bias variability and symptoms of posttraumatic stress disorder. J Trauma Stress 2014; 27: 232-239.

[19] Khan AM et al. Cognitive behavioral therapy versus eye movement desensitization and reprocessing in patients with post-traumatic stress disorder. Cureus 2018; 10(9): e3250.
[20] Knudsen El. Instructed learning in the auditory localization pathway of the barn owl. Nature 2002; 417: 322-328.

[21] Knudsen El. Neural circuits that mediate selective attention: A comparative perspective. TINS 2018; 41: 789-805.

[22] Krauzlis RJ, Lovejoy LP, Zénon A. Superior colliculus and visual spatial attention. Annu Rev Neurosci 2013; 36: 165-182.

[23] Landin-Romero $R$ et al. How does eye movement desensitization and reprocessing therapy work? A systematic review on suggested mechanisms of action. Front Psychol 2018; 9: 1395.

[24] Mollica RF et al. Dose-effect relationships of trauma to symptoms of depression and post-traumatic stress disorder among Cambodian survivors of mass violence. British Journal of Psychiatry 1998; 173: 482-488.

[25] Mollica RF et al. The dose-effect relationships between torture and Ppychiatric symptoms in Vietnamese ex-political detainees and a comparison group. Journal of Nervous \& Mental Disease 1998; 186(9): 543-553.

[26] Müller JR et al. Microstimulation of the superior colliculus focuses attention without moving the eyes. PNAS 2005; 201: 524-529.

[27] Naim R et al. Threat-related attention bias variability and post traumatic stress. Am J Psychiatry 2019; in press.

[28] Pouille F et al. Input normalization by global feedforward inhibition expands cortical dynamic range. Nature Neuroscience 2009; 12 : 1577-1585.

[29] Ray S, Mounsell JHR. Do gamma oscillations play a role in cerebral cortex? TICS 2015; 19: 78-85.

[30] Reynolds JH, Heeger D].The normalization model of attention. Neuron 2009; 61: 168-185.

[31] Schweitzer RD et al. Recently resettled refugee women-at-risk in Australia evidence high levels of psychiatric symptoms: individual, trauma and post-migration factors predict outcomes. BMC Med 2018; 16: 149.

[32] Senn $V$ et al. Long-range connectivity defines behavioral specificity of amygdala neurons. Neuron 2014; 81(2): 428-37.

[33] Servan-Schreiber D et al. Eye Movement Desensitization and Reprocessing for Posttraumatic Stress Disorder: A Pilot Blinded, Randomized Study of Stimulation Type. Psychother Psychosom 2006; 75: 290-297.

[34] Spitzer M. Normierung im Gehirn. Nervenheilkunde 2007; 26: 200-202.

[35] Spitzer M. Gedächtnisspuren löschen? Auf das Timing kommt es an! Nervenheilkunde 2012; 31: 944-948.

[36] Tinghög P et al. Prevalence of mental ill health, traumas and postmigration stress among refugees from Syria resettled in Sweden after 2011: a populationbased survey. BMJ Open 2017; 7: e018899.

[37] Turrigiano G.Too many cooks? Intrinsic and synaptic homeostatic mechanisms in cortical circuit refinement. Annu Rev Neurosci 2011; 89-103.

[38] White B] et al. Superior colliculus neurons encode a visual saliency map during free viewing of natural dynamic video. Nat Commun 2017; 8: 14263.

[39] White BJ et al. Superior colliculus encodes visual saliency before the primary visual cortex. PNAS 2017; 114: 9451-9456.

[40] Wilson G et al. The Use of Eye-Movement Desensitization Reprocessing (EMDR) Therapy in Treating Post-traumatic Stress Disorder-A Systematic Narrative Review. Front Psychol 2018; 9: 923.

[41] Vokoun CR et al. Response normalization in the superficial layers of the superior colliculus as a possible mechanism for saccadic averaging. The Journal of Neuroscience 2014; 34: 7976-7987.

[42] Badura-Brack AS et al. Effect of attention training on attention bias variability and PTSD symptoms: Randomized controlled trials in Israeli and U.S. Combat Veterans. Am J Psychiatry 2015; 172: 1233-1241. 